\title{
A Finite Element Method Application for Nonlinear Mechanical Response of Three-Dimensional Beams
}

\author{
Hakan Tanriöver* \\ Çankaya University, Mechanical Engineering Department, Ankara, Turkey
}

*Corresponding Author: Hakan Tanrı̈ver, Cankaya University, Mechanical Engineering Department, Ankara, Turkey

\begin{abstract}
Mechanical behavior of beams under large rotations and displacements was investigated. Using co-rotational approach given by Crisfield, three-dimensional beam finite element was modeled in Mathematica environment. The yielding non-linear equation system was solved by utilizing Newton-Raphson technique. Dynamic balance equations and numerical time integration method were introduced, while the solution process was left for a future work. Comparisons with the results of ANSYS and other works in the literature are accomplished.
\end{abstract}

Keywords: Three-Dimensional Beams, Co-Rotational, Mathematica, Geometric Nonlinear

\section{INTRODUCTION}

In the three-dimensional (3D) beam models for flexible mechanisms, robotic arms, springs, space truss systems and similar structures, large displacements and rotations must be considered. One of the major difficulties here is that the rotational variables do not have a vectorial behavior [1]. Some researchers used a co-rotational formulation which decomposes the displacements resulting from the rotations and the deformations. In this formulation, there is an undeforming coordinate system that is attached to the element and rotating with it.

The initial research works modeling beam elements using a co-rotational approach were carried out by Oran and Kassimali [2], and Belytschko et al. [3]. Oran and Kassimali solved the dynamic behavior of two-dimensional beams utilizing beam-column type method with co-rotational formulation. In their work, they have obtained the tangent stiffness matrix for small deformations but did not take into account the large rotations. The tangent stiffness matrices and nonlinear analyses of 3D beams under small deformations and large rotations have been given by some of the researchers for co-rotational formulation [3-5]. Pai et al. performed a large deformation analysis of 3D beams using totalLagrangian displacement based finite element formulation which accounts for geometric nonlinearities and initial curvatures [6]. Le et al. investigated the dynamic behavior of 3D beam elements using Newmark time stepping methods along with a co-rotational formulation for finite elements [7]. In their work, different methods were tested and Crisfield's co-rotational formulation [4] is found to be the best alternative. Jonker et al. proposed a geometrically nonlinear solution for 3D beams having a shear deformable Timoshenko finite element model [8]. Other research works on 3D beam formulations can also be found in the literature [9-14].

In this paper the co-rotational formulation of Crisfield is utilized for small strain, large displacement and rotation behavior of $3 \mathrm{D}$ beams. Using the aforementioned formulation, a finite element code is developed in Mathematica environment. The Mathematica code is not given in this paper because of its length, however it can be provided upon request. Some numerical examples from the literature are handled and the results are given. Also an ANSYS finite element analysis is performed and a comparison with the results is presented.

\section{EQUATIONS OF EQUILIBRIUM AND FORMULATION}

Using Crisfield formulation, we can obtain the following equations of equilibrium for 3D beam under small strain and large rotations and displacements. 

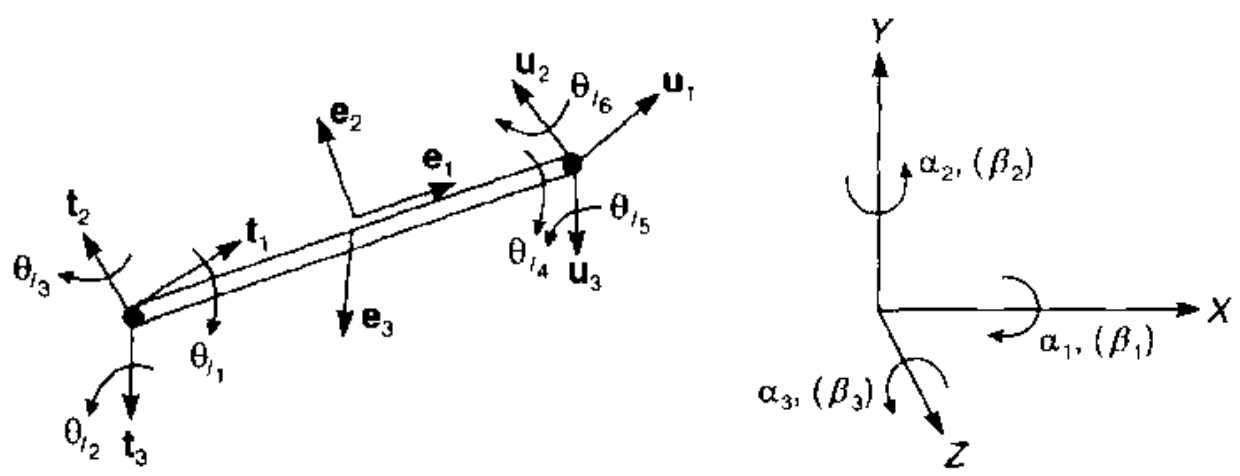

Figure1. Beam element and degrees offreeedom [4].

The finite element model has three rotational and three translational degrees of freedom for each node. Local coordinate system and the corresponding degrees of freedoms are shown in Figure-1. In the equations subscript "l" denotes for the local variables. Small strain assumption hence a local linear response is enforced through the element, while the global response of the beam is nonlinear due to the large rotations.

Local degrees of freedom for the element are

$P_{l}^{T}=\left(d_{11}^{T}, \theta_{11}^{T}, d_{12}^{T}, \theta_{12}^{T}\right)$

$\mathrm{d}_{l}^{T}=\left(\mathrm{u}_{11}, v_{11}, w_{12}\right)$

The unit vector $\mathbf{e}_{1}$ is defined on the direction passing through the first and second nodes of the element and can be calculated as

$\mathbf{e}_{1}=\left(\mathrm{x}_{21}+\mathbf{d}_{21}\right) / l_{2}$

A linear relationship between the nodal forces $\left(\mathbf{q}_{l}\right)$ and rotations $\left(\mathbf{p}_{l}\right)$ can be written based on EulerBernoulli beam theory.

$\mathbf{q}_{l}=\mathbf{K}_{l} \mathbf{p}_{l}$

Axial forces $(\boldsymbol{N})$ and moments $(\mathbf{M})$ in the beam can be given as

$\boldsymbol{N}=\frac{E A}{l_{0}} u_{l}, \quad \mathbf{M}=\mathbf{D}\left(\boldsymbol{\theta}_{l}-\boldsymbol{\theta}_{l 0}\right)$

Here, the subscript zero denotes for the initial position and $\mathbf{D}$ relates the moments to the slopes. Assume that there is a relation such as $\delta \mathbf{p}_{l}=\mathbf{F} \delta \mathbf{p}$ between the local and global degrees of freedom, equating the virtual works in local and global systems one can write

$\mathbf{q}_{i}=\mathbf{F}^{\mathrm{T}} \mathbf{q}_{l i}=\mathbf{F}^{\mathrm{T}} \mathbf{K}_{l} \mathbf{p}_{l}$

and find the general internal force vector $\mathbf{q}_{i}$. Using this the tangent stiffness matrix can be obtained.

$\delta \mathbf{q}_{i}=\mathbf{F}^{\mathrm{T}} \delta \mathbf{q}_{l i}+\delta \mathbf{F}^{\mathrm{T}} \mathbf{q}_{l i}=\mathbf{F}^{\mathrm{T}} \mathbf{K}_{l} \mathbf{F} \delta \mathbf{p}+\mathbf{K}_{t \sigma} \delta \mathbf{p}$

The unit vectors defined for an element can be seen in Figure-2. Here, the rotation matrices E, $U$ and $\mathrm{T}$ are constructed by writing the corresponding unit vectors as a column. The detailed information about obtaining the tangent stiffness matrix can be seen in Reference [4].

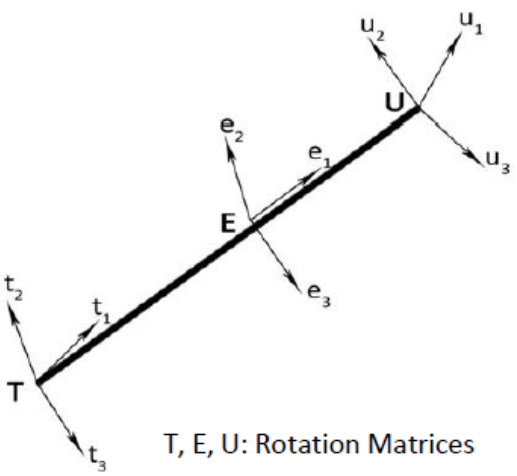

Figure2. Local unit vectors in the element. 
The tangent stiffness matrix is not symmetric due to its construction method. However, the matrix tends to be symmetric as the iteration process proceeds to the equilibrium point. At this point artificial methods to obtain a symmetric matrix can be used. The governing nonlinear equation systems are solved by using the Newton-Raphson method.

The dynamic behavior of the beam can be resolved by utilizing a time stepping scheme. In general, the dynamic equilibrium equations can be briefed as

$$
\left(\mathbf{q}_{i, n+1}-\mathbf{q}_{e, n+1}\right)+\mathbf{M} \ddot{\mathbf{d}}_{n+1}+\mathbf{C} \dot{\mathbf{d}}_{n+1}=\mathbf{0}
$$

Here, the subscripts $i$ and $e$ denotes for the internal and external force vectors, respectively. $\mathbf{M}, \mathbf{C}$ and d are the mass, damping and displacement vectors, respectively. For time stepping, Newmark- $\beta$ time integration method can be used, in this case the displacement and velocity vectors will be

$\mathbf{d}_{n+1}=\mathbf{d}_{n}+\Delta t \dot{\mathbf{d}}_{n}+\Delta t^{2}\left((1-2 \beta) \ddot{\mathbf{d}}_{n}+2 \beta \ddot{\mathbf{d}}_{n+1}\right) / 2$

$\dot{\mathbf{d}}_{n+1}=\dot{\mathbf{d}}_{n}+\Delta t\left((1-\gamma) \ddot{\mathbf{d}}_{n}+\gamma \ddot{\mathbf{d}}_{n+1}\right.$

These equations can be substituted into the dynamic equilibrium equations and using the tangent stiffness matrix

$\mathbf{q}_{i, n}-\mathbf{q}_{e, n+1}+\mathbf{K}_{t, n} \Delta \mathbf{d}+\mathbf{M}\left(\frac{\mathbf{4}}{\Delta t^{2}} \Delta \mathbf{d}-\frac{\mathbf{4}}{\Delta t} \dot{\mathbf{d}}_{n}-\ddot{\mathbf{d}}_{n}\right)+\mathbf{C}\left(\frac{\mathbf{2}}{\Delta t} \Delta \mathbf{d}-\dot{\mathbf{d}}_{n}\right)=\mathbf{0}$

can be written. Hence, in each time step the nonlinear equations can be solved using the NewtonRaphson method. The equation systems for dynamic problems are explained here briefly, while the dynamic analysis is left for the future work. The numerical examples given below are static cases.

\section{NUMERICAL EXAMPLES}

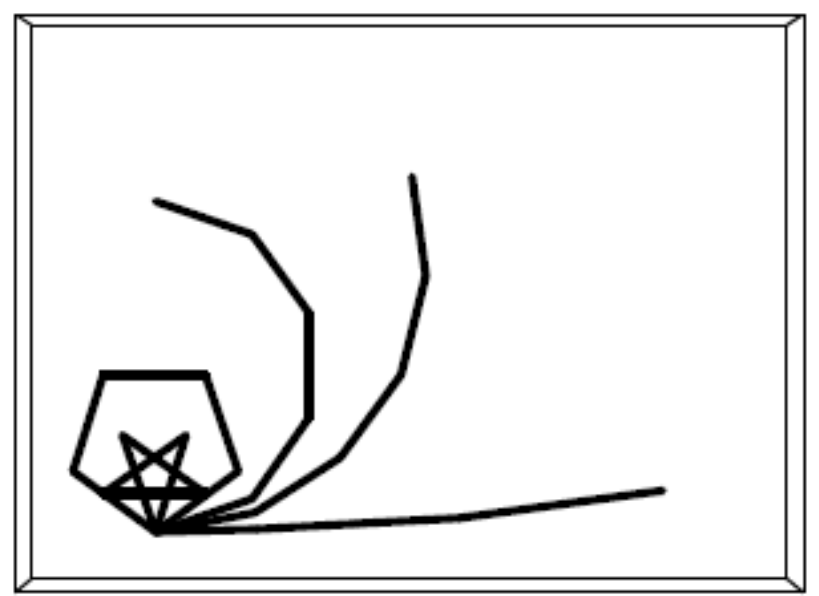

Figure3. Cantilever beam subject to endmoment. The deformation of the beamfor different moments can be seen in the same graph. The moment values in increasing form are: $0.025 M^{*}, 0.3 M^{*}, 0.5 M^{*}, M^{*}, 2 M^{*} . M^{*}=\frac{M L}{2 \pi E I}$

\section{Case 1. Cantilever Beam Subject to End Moment}

The left end of the beam has a cantilever support and a moment is applied from the right end (Figure 3). This problem can be solved using two dimensional elements and it is a pure bending problem. However, it is solved using the developed Mathematica code and Crisfield's beam formulation. In the finite element model there are only five elements and the convergence criteria for Newton-Raphson method is taken as $\epsilon=\frac{\|\mathbf{g}\|}{\left\|\mathbf{q}_{e}\right\|}<1 / 1000$. Here $\mathbf{g}$ is the unbalanced force vector and $\mathbf{q}_{\mathrm{e}}$ is the external force vector. In each loading step 4 iterations are required.

\section{Case2. Forty-Five Degree Bend}

The bend is fixed at the origin and it is subjected to a out of plane load at the other end (see Figure-4). The bend has a unit cross-sectional area and an elasticity modulus of $10^{7}$. The finite element model in Mathematica has 8 elements and 9 nodes. The maximum load value is 600 and it is applied in 8 load steps. In each load step 5 iterations were found to be enough to satisfy the convergence criteria. The deformed shapes of the bend under different load steps are shown in Figure-5. 


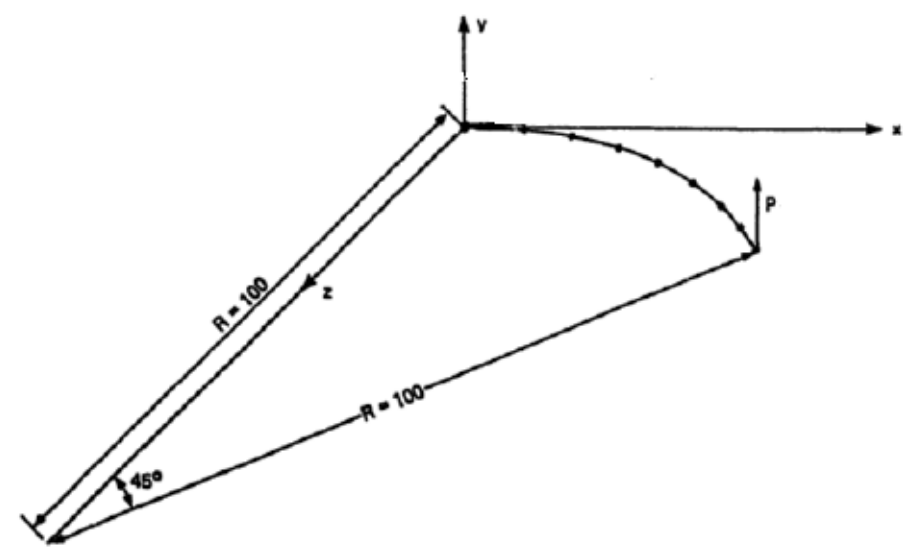

Figure4. Geometry of the forty-five degree bend [4].

To make a comparison the same problem was handled using commercial finite element package ANSYS. In ANSYS analysis the beam is modeled using 8 BEAM188 type elements with 17 nodes (see Figure-6). In the nonlinear solution process ANSYS used 3 load steps and 7 iterations in each step. In order to understand the nonlinear geometric effect, both linear and nonlinear analyses are performed. The results of ANSYS and Mathematica code are presented in Table-1. Results of the other researchers can be seen in the Reference [4]. A very good agreement can be seen for the nonlinear results. The effect of geometric nonlinearity is obvious in this case and must not be neglected.

Table1. Out of plane displacement of the end point of the $45^{\circ}$ bend. Lin.: Linear. N.Lin.: Nonlinear.

\begin{tabular}{|c|c|c|c|}
\hline Load & ANSYS (Lin.) & ANSYS (N.Lin.) & Mathematica \\
\hline 300 & 58.837 & 40.923 & 40.533 \\
\hline 450 & 88.256 & 48.997 & 48.788 \\
\hline 600 & 117.670 & 53.785 & 53.710 \\
\hline
\end{tabular}

\section{Case 3. Cantile ver Helical Spring}

Here a helical spring which is fixed at the bottom end and under a concentrated force at the opposite end is modeled by using 20 elements in the Mathematica code (see Figure-7). Both linear and nonlinear responses are considered, and the displacements of the tip point are given below. The elasticity modulus $(E)$, radius of the helix $(R)$, spring diameter $(d)$, pitch angle $(p)$ and the applied force $(P)$ are taken as; $E=10^{7}, R=10, d=1, p=10^{0}, P=100$. The displacement components $\left(u_{i}\right)$ at the tip point are given in Table-2. In the nonlinear analysis only one load step is used and 7 iterations were required.

Table2. Displacement components at the tip point of helical spring.

\begin{tabular}{|c|c|c|c|}
\hline Type/Displacements & $\boldsymbol{u}_{\boldsymbol{x}}$ & $\boldsymbol{u}_{\boldsymbol{y}}$ & $\boldsymbol{u}_{\boldsymbol{z}}$ \\
\hline Lin. & -0.821422 & 0.37414 & 3.12548 \\
\hline N.Lin & -0.921413 & 0.35634 & 2.97771 \\
\hline
\end{tabular}

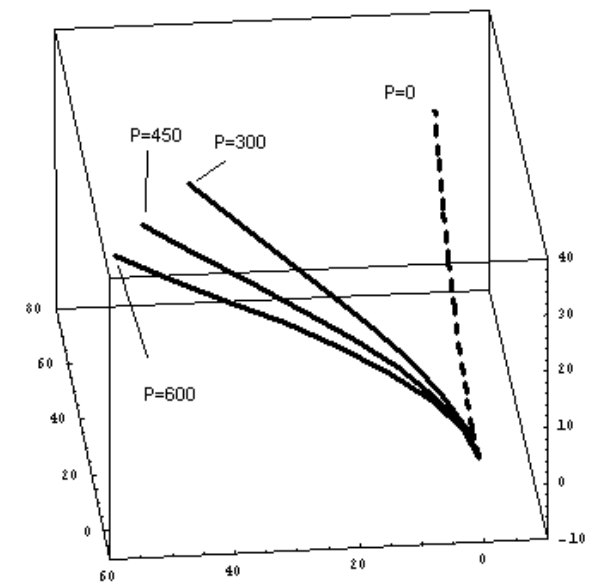

Figure5. Initial and deformed geometries for $45^{\circ}$ bend (Mathematica). 


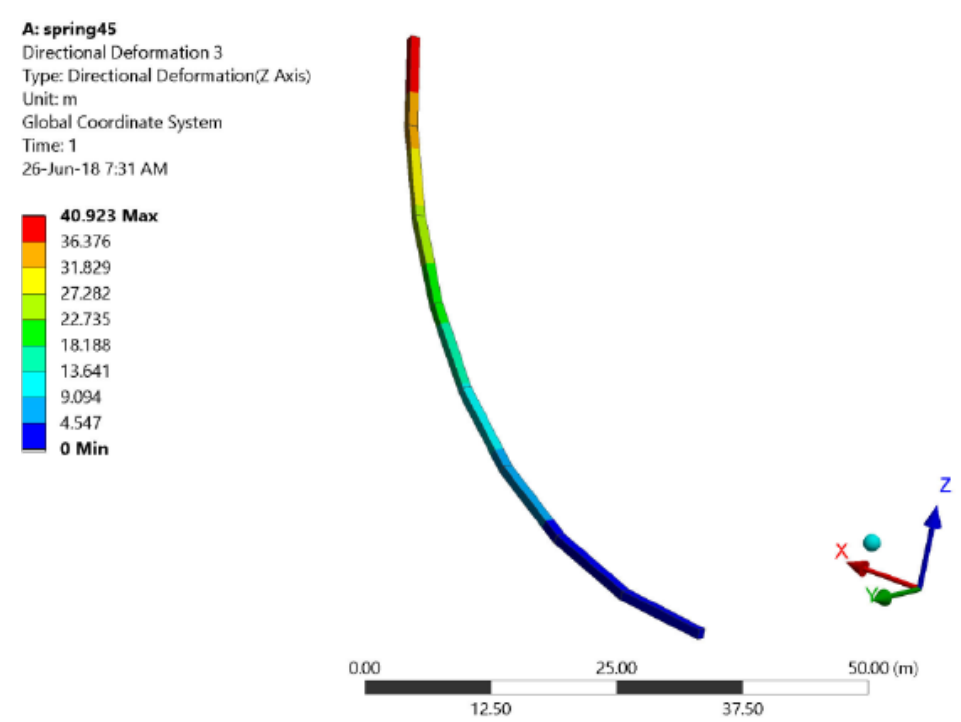

Figure6. Deformed geometry of $45^{\circ}$ bend under the load of 300 units (ANSYS).

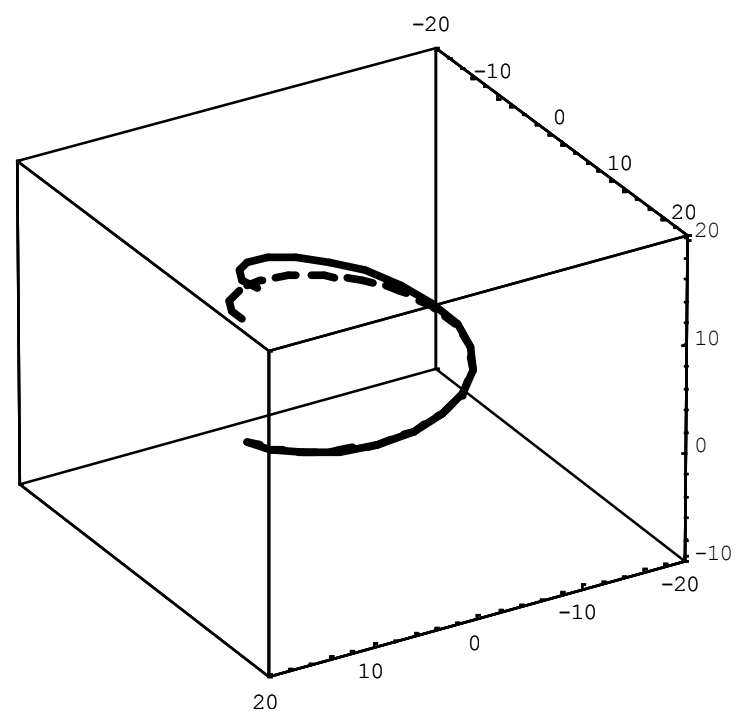

Figure7. Deformed and undeformed geometry of the cantilever coil spring.

\section{CONCLUSion}

Using Crisfield's co-rotational formulation which takes into account large displacements and rotations, mechanical behavior of 3D beams under static loads was investigated. The beam finite element modeling was accomplished in Mathematica environment. The governing nonlinear equation system was solved with the help of the Newton-Raphson method. Three different numerical cases are selected and the results are compared with those of the literature and the commercial finite element program ANSYS and a very close agreement is observed. Dynamic behavior equations are obtained and for the time integration Newmark- $\beta$ method is proposed for the future work. Note that all the formulations are symbolic in the Mathematica program and it is very easy to develop and modify a code. Variable parameters can be set in the code, and it is possible to integrate an optimization code to perform a structural optimization problem.

\section{REFERENCES}

[1] Argyris, J., An excursion into large rotations, Comp. Methods Appl. Mech. Engrg., 32, pp. 85-155, (1982).

[2] Oran, C. and Kassımali, A., Large deformations of framed structures under static and dynamic loads, Computers \& Structures, 6, pp. 539-547, (1976).

[3] Belytschko, T., Large displacement, transient analysis of space frames, Int. J. for Num. Meth. in Engrg., 11, pp. 65-84, (1977).

[4] Crisfield, M.A., A consistent co-rotational formulation for non-linear, three-dimensional, beam-elements, Comp. Methods Appl. Mech. Engrg., 81, pp. 131-150, (1990). 
[5] Hsiao, K.M., Lin, J., Y., Lin,W. Y., A consistent co-rotational finite element formulation for geometrically nonlinear analysis of 3-D beams, Comp. Methods Appl. Mech. Engrg., 169, pp. 1-18, (1999).

[6] Pai, P.F., Large deformation tests and total-Lagrangian finite-element analyses of flexible beams, Int. J. of Solids and Structures, 37, pp. 2951-2980, (2000).

[7] Le, T.-N., Battini, J.-M., Hijiaj, M., Dynamics of 3D beam elements in a corotational context: A comparative study of established and new formulations, Finite Elements in Analysis and Design, 61, pp. 97-111, (2012).

[8] Jonker, J.B., A geometrically nonlinear formulation of three-dimensional beam element for solving large deflection multibody system problems, International Journal of Non-Linear Mechanics, 53, pp.63-74, (2013).

[9] Bathe, K.-J., Large displacement analysis of three-dimensional beam structures, Int. J. For Num. Meth. in Engrg., 14, pp.961-986, (1979).

[10] Simo, J.C., A finite strain beam formulation. The three dimensional dynamic problem, Part-I, Comp. Methods Appl. Mech. Engrg., 49, pp. 55-70, (1985).

[11] Rhim, J. and Lee, S. W., A vectorial approach to computational modelling of beams undergoing finite rotations, Int. J. for Num. Meth. in Engrg., 41, pp. 527-540, (1998).

[12] Le, T.-N., Battini, J.-M., Hijiaj, M., A consistent 3D corotational beam element for nonlinear dynamic analysis of flexible structures, Comp. Methods Appl. Mech. Engrg, 269, pp. 538-65, (2014).

[13] de Miranda, S., Madeo, A., Melchionda, D., Patruno, L. ve Ruggerini, A.W., A corotational based geometrically nonlinear generalized beam theory: buckling FE analysis, Int. J. Solids and Structures, 121, pp. 212-227, (2017).

[14] Cho, H., Kim, H., Shin, S., Geometrically nonlinear dynamic formulation for three-dimensional corotational solid elements, Comput. Meth. Appl. Mech. Engrg., 323, pp. 301-320, (2018).

\section{AUTHORS' B IOGRAPHY}

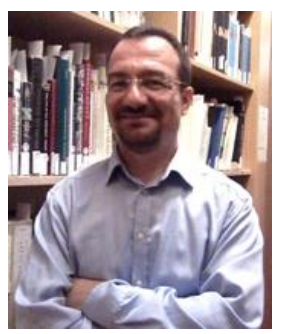

Hakan Tanriöver, is an Assistant Professor at the Mechanical Engineering Department of Çankaya University, Turkey. He has published journal articles and conference papers in the areas of mechanics of materials and structures. His other research interests include finite element methods, nonlinear mechanics and mechanics of energy storage materials. He is a member of MRS.

Citation: Hakan Tanriöver. (2018)" A Finite Element Method Application for Nonlinear Mechanical Response of Three-Dimensional Beams", International Journal of Modern Studies in Mechanical Engineering, 4(2), pp.24-29. DOI: http://dx.doi. org/10.20431/2454-9711.0402004

Copyright: (C) 2018 Hakan Tanriöver, This is an open-access article distributed under the terms of the Creative Commons Attribution License, which permits unrestricted use, distribution, and reproduction in any medium, provided the original author and source are credited. 